\title{
Discursive work in resisting stereotypic representations of the Chinese among Chinese students
}

\begin{abstract}
The negative effects of stereotyping arising from a victim's acceptance and internalisation of stereotype identities are well-known. As stereotypes are created and maintained in discourse, understanding how targets of stereotyping employ discursive resources to resist the constraining structures of stereotypic identities imposed upon them can provide insight into the process of stereotyping and contribute to efforts to reduce the threat of stereotyping. We examined the strategies used by targets of stereotyping in contesting stereotypic representations of their social group through the mobilisation of a range of discourse strategies when presented with stereotyping attacks on the group. The findings revealed that stereotypes are subtle in nature and may not be easily recognised and hence, difficult to resist. Participants employed a number of discourse strategies to repair their fragmented self and group identities. However, in their attempt to maintain identity coherence, they ended up using stereotyping discourses themselves to devalue the perceived outgroup as well as subgroups they created within their own social group. The study highlights the complexity of stereotyping and its self-perpetuating character, and sheds light on the difficulty faced by targets of stereotyping discourse in reconciling their identities through intense discursive and identity work.
\end{abstract}

Keyword: Discourse studies; Stereotyping discourse; Contesting stereotyping identities; Discourse strategies; Identity work; Chinese identity; Chinese stereotype 